lanceolata Keller. Serratur und schwache, aber auch auf die Costa übergehende, später verschwindende Behaarung der Petiolen, die der lanceolata Opiz auch deren Foliolen, - aber Blüthenzweige bestachelt, Pedunkeln schwach befläumt. Receptakeln kleiner, schmäler, ovoid, Griffel kahl, selten mit 1-2 Härchen, also weder lanceolata typica, noch decalvata Crép., sondern zweifelsohne ein Mischling der am Fusse des Hádyberges bäufig und nebeneinander vorkommenden zu zwei differenten Subsectionen gehörenden $\boldsymbol{R}$. levistyla Rip. und $\boldsymbol{R}$. lanceolata Opiz. Ketler.

Im Nachhange zu den vorstehend aufgezählten natürlichen $\mathrm{Ab}$ änderungen sei hingegen einer Varietät gedacht, die im Laube, Umriss der Foliolen ganz mit jenen der Rosa Chaboissei Gren. nach Exemplaren aus Crépin's Hand determ. Déséglise übereinstimmt, aber stylis "obscure pilosis" receptaculis floriferis ovoideo-oblongis aut obovoideo-oblongis, also im Sinne Déséglise's (conf. dessen Catalogue pag. 155) diese Abänderung innerhalb des Formenkreises der $\boldsymbol{R}$. oblonga Déségl, et Rip. aufzuzählon kommt; dann einer sehr seltenen, besonders schönen Form der $\boldsymbol{R}$. sphaeroidea Rip., deren rami conferti, floriferi breves et aculeati; stipulae supra, sepala utrinque purpurascentia rami bornotini virides; foliola orbicularia aut (superiora) obovato-rotundata, breviuscula, parva ant mediocria; fructus ample subglobosus. Eine auffallende und nach der massenhaften Aufsammlung Dr. Formánek's seltene Form, im Laube von der Forne an die $\boldsymbol{R}$. armatissima $R$. et $\mathrm{D}$. erinnernd, und vou dor bereits benannten und gekennzeichneten var. latistipula Gdgr. Tab. 1779 nur in den mehr bestachelten Zweigen abweichend, daher wir ihr $\theta$ Neubenennung unterlassen. Keller.

(Schluss folgt.)

\title{
Berichtigung einiger die böhmische Flora betreffenden Angaben in Dr. E. Roth's "Additamenta".
}

Von Dr. Lad. Čelakovský.

In den jüngst erschienenen "Additamenta ad Conspectum Florae europeae editum a Cl. C. F. Nyman" von Dr. E. Roth werden mehrere Arten irrthümlich als in Böhmen wachsend angegeben und zwar: Erysimum lanceolatum R. Br., Polygala major Jacq., Silene rupestris L., Hacquetia epipactis DC., Inula ensifolia L., Hieracium sabinum Seb. et Mauri, Teucrium montanum L., Epipactis microphylla Sw. Da der Verf. der Additamenta laut Vorrede seine nachträglichen Augaben auf Grund von Exsiccaten, die sich in Berliner Herbarien betinden, gemacht hat, so dürfte man daraus schliessen, dass die böhmischen Floristen von einer Reihe von böhmischen Arten keine Kenntniss haben, für welche sich selbst in Berlin Belege 
finden. Ueber meine directe Anfrage war jedoch Herr Roth so gefällig, mir die schriftliche Erklärung zu geben, dass die meisten dieser Angaben auf einer irrigen Auffassung der Scheden nicht böhmischer (zumeist ungarischer) Pflanzen und eine auf unrichtiger $\mathrm{Be}-$ stimmung beruht. Obzwar Hr. Roth diess in Just's Botanischem Jahresbericht berichtigen will, glaubte ich diess auch österreichischen Leserkreisen in diesen Blättern bekannt geben zu sollen, damit nicht weitere irrthümliche Meinungen daraus entstehen.

Der Verfasser der Additamenta wäre auf seinen Irrthum in Bezug auf die obigen Arten aufmerksam geworden und hätte ihn gewiss vermieden, wenn er einmal den Prodromus der Flora Böhmens und die Nachträge zu demselben (die als "Resultate der botanischen Durchforschung Böhmens" bis 1884 fortgesetzt sind) nachgeschlagen hätte.

Hieran mögen noch einige andere berichtigende Bemerkungen geknüpft werden.

Adonis autumnalis L. und Linaria genistaefolia L. hätten den Zusatz Bohemia in den Additamentis auch nicht verdient, denn beide sind nur ganz eiazeln und vorübergehend, wohl als Gartenflüchtlinge oder sonst eingeschleppt, in Böhmen gefunden worden. Ebensowenig ist Colutea arborescens $\mathrm{L}$. in Böhmen wirklich einheimisch, wenn sie auch, gleich Syringa vulgaris, öfter im Freien gepflanzt wird und quasi verwildert.

Noch sind mir einige Irrthümer aufgefallèn. Silene italica Pers. und Silene nemoralis W. $K$. erhielten in den "Additamenta" beide den Zusatz Bohemia. Es wächst bei uns aber nur eine Form, nämlich die letztere; die erstere nur insofern, als die letztere als Abart $\mathrm{zu}$ ihr gezogen wird. Trifolium elegans Savi wächst nicht in Böhmen, sondern nur eine andere ähnliche Form von $T$. hybridum L., ( $\beta$. parviflorum Cel.), was auch aus meinem Prodromus zu ersehen gewesen wäre. Auch Festuca duriuscula L. Sp. pl. (teste Hackel), Nyman Consp. p. 829, darf nicht aus Böhmen angegeben werden, da die Art, die bei uns seit alters als $F$. duriuscula L. ausgegeben wird, die $F$. duriuscula Host $=F$. sulcata Hackel, Nyman Consp. p. 828 ist, zu welcher also der Zusatz Bohemia gehört hätte. Auch das ist in den Nachträgen zum Prodr. Fl. Böhm. zu lesen. Dessgleichen ist "Hieracium juranum Fr." $\theta$ Bohemia (i. $\theta$. Sudetorum) richtiger Hier. corconticum Knaf nebst $H$. asperulum Freyn, aber nicht das westliche $\boldsymbol{H}$. juranum oder $\boldsymbol{H}$. jurassicum Gris., worüber mein Artikel in der Oesterr. Botan. Zeitschr. hätte Auskunft geben können.

Im Vorwort versichert Herr Roth: „Die italienischen Inseln, Polen, Böhmen etc. habe ich stets namhaft gemacht, wo Nyman manchmal diese Länder angibt, manchmal fortlässt."

Es ist nun allerdings in Nyman's Conspectus ein Uebelstand, dass daselbst die Bezeichnung "Austria" in verschiedenem Sinne gebraucht wird, bald speciell für das Erzherzogthum Oesterreich, bald, wenn die Art allgemeiner verbreitet ist, für eine grössere Län- 
dergruppe des österreichischen Staates (zumeist für die „cisleithanische" Hälfte), welcher Unterschied nicht ersichtlich gemacht ist. Dieser Unbestimmtheit und Zweidentigkeit wollte Herr Roth, was nur zu billigen ist, in der oben angezeigten Weise abhelfen. Dann hätte diess aber mit aller möglichen Vollständigkeit durchgeführt werden sollen. Das ist aber keineswegs geschehen, wie ich beispielsweise nur für die erste Familie Ranunculaceae zeigen will.

Nach dem erklärten Grundsatz des Verfassers der Additamenta sollten auch Clematis recta, Thalictrum aquilegifolium, angustifolium simplex, Pulsatilla vernalis, Hepatica triloba, Ranunculus nemorosus, cassubicus, Batrachium confusum (Ran. Petiveri Koch), Isopyrum thalictroides, Trollius europaeus, Aconitum lycoctonum, A. napellus den Zusatz Bohemia erhalten. Wir vermisses ihn aber hier überall. Jetzt ist die Sache noch schlimmer als in Nyman's Conspectus geworden, denn jetzt muss Derjenige, der der Vorrede zu den Additamenta Glauben schenkt, wenn er nicht anderweitig besser informirt ist, annehmen, dass alle die genannten Ranunculaceen in Böhmen gar nicht wachsen. Mit den übrigen Pflanzenfamilien verhält es sich ebenso, woraus zu entnehmen, in wie vielen anderen Fällen die Additamenta den Nachschlagenden im Stiche lassen müssen.

H. Roth hat offenbar den unrechten Weg zu seinem Ziele eingeschlagen, indem er wieder nur die in Berliner Herbarien zufällig befindlichen Exsiccaten berücksichtigte, den Prodromus der Fl. Böhmens sammt Nachträgen, den er, um etwas Vollständiges zu liefern, in erster Reihe hätte excerpiren müssen, ganz bei Seite liess.

Ich beabsichtigte hier nur in die Behandlung der böhmischen Flora in den "Additamenta" einzugehen; bemerke aber nur noch per parenthesin, dass eine Callitriche "pentacanthum Hayn." nicht existirt, wohl aber ein Ceratophyllum pentacanthum Hayn., und dass die Orobanche ionantha Kern. so gut wie die O. Muteli F. Sch. eine Phelipaea ist.

\section{Zur Verbreitung und Teratologie von Typha und Sparganium.}

\section{Von Dr. Vincenz v. Borbáis.}

1. Typha latifolia L. ist in Ungarn nicht selten, sie wächst aber manchmal auch mit $T$. Shouttleworthii zusammen. - Var. ambigua Sond. boi Vésztö.

Bei Vésztó und Nagy-Enyed fand ich Exemplare, bei welchen die Spitze des fruchttragenden Stengels gabelig gespalten war und je ein Gabelast einen Fruchtkolben trug. Sie standen dicht beisammen oder divergirten an der Spitze und erschienen als Zwillinge. Die einander berührenden Seiten der beiden Blüthenstände waren ganz nor- 